\title{
Bezafibrato em lactente portador de lipodistrofia generalizada congênita e hipertrigliceridemia grave
}

\author{
Bezafibrate in an infant with congenital generalized \\ lipodystrophy and severe hypertriglyceridemia
}

Rogério Santiago Araújo', André de Paula Silva Ramos²,

Máriton de Araújo Sousa Borges ${ }^{3}$

\begin{abstract}
SUMÁRIO
Lipodistrofia congênita generalizada (CGL) com hipertrigliceridemia extrema desde o primeiro ano de vida está associada a piores riscos metabólicos. Foram utilizados dados contidos no prontuário do paciente, bem como revisão bibliográfica para composição do texto. Relatamos o caso de um lactente com fenótipo típico e hipertrigliceridemia de $1.360 \mathrm{mg} / \mathrm{dL}$, que foi tratado com bezafibrato na dose de 30 a $60 \mathrm{mg} / \mathrm{dia}$ dos 11 meses aos 5 anos e 6 meses de idade, aferindo um nadir de triglicérides de $55 \mathrm{mg} / \mathrm{dL}$. Evolução clínico-laboratorial antes e após bezafibrato foi feita ao longo de cinco anos e seis meses. O fenótipo apresentado foi classificado clinicamente em CGL tipo 2. Apesar do controle eficiente da hipetrigliceridemia e da ausência de desenvolvimento de diabetes melito, o uso de bezafibrato não impediu o aparecimento de esteatose hepática durante a evolução. A terapia antilipemiante com fibrato se mostrou eficaz em manter níveis normais de triglicerídeos, colesterol e suas frações e não se associou a efeitos colaterais graves durante o período descrito. Arq Bras Endocrino Metab. 2013;57(8):653-8
\end{abstract}

\section{SUMMARY}

Congenital generalized lipodystrophy (CGL) with severe hypertriglyceridemia in a children less than 1 year of age is associated with worse metabolic risk. We used data from patient records, as well as extensive literature research to write the manuscript. We report the case of an infant with typical phenotype of CGL and hypertriglyceridemia of $1,360 \mathrm{mg} / \mathrm{dL}$ who was treated with bezafibrate at a dose of 30 to $60 \mathrm{mg} /$ day from age 11 months to 5.5 years old, with a measurement of nadir of triglycerides of $55 \mathrm{mg} / \mathrm{dL}$. Clinical evolution and clinical laboratory tests before and after bezafibrate were carried out over 5 years and 6 months. Phenotype was classified as CGL type 2. Despite the efficient control of hypertriglyceridemia and absence of development of diabetes mellitus, the use of bezafibrate did not prevent the onset of hepatic steatosis during evolution. Hypolipidemic therapy with bezafibrate proved effective in maintaining the levels of triglycerides, cholesterol and its fractions at normal levels, and its use was not correlated with severe side effects during the described period. Arq Bras Endocrinol Metab. 2013;57(8):653-8
${ }^{1}$ Disciplina de Endocrinologia, Centro de Ciências da Saúde, Universidade Estadual do Piauí (UESPI), Teresina, PI, Brasil ${ }^{2}$ Centro de Ciências da Saúde, UESPI, Teresina, PI, Brasil

${ }^{3}$ Centro de Ciências da Saúde, Universidade Federal do Piauí (UFPI), Teresina, PI, Brasil
Correspondência para: Rogério Santiago Araújo Rua Governador Artur de Vasconcelos, 670, Centro Sul Teresina, PI, Brasil dr_rogeriosantiagoaraujo@hotmail. com

Recebido em 2/Jul/2012 Aceito em 27/Maio/2013

\section{INTRODUÇÃO}

$\mathrm{L}$ ipodistrofia generalizada congênita (CGL, congenital generalized lipodystrophy), também conhecida como síndrome de Berardinelli-Seip (BSCL, MIM \#269700), é uma doença autossômica recessiva caracterizada por graus variáveis de redução de gordura nos tecidos adiposos (1). A intensidade de perda de gordura corporal determina a severidade do comprometimento metabólico e outras complicações $(2,3)$. Na última década, considerável progresso ocorreu na elucidação da base molecular das lipodistrofias gené- ticas, relacionando-as a pelo menos 11 loci gênicos, sendo quatro deles (AGPAT2, BSCL2, CAV1 e PTRF) relacionados especificamente à CGL (4). A prevalência da CGL é baixa, de aproximadamente 1:10.000.000 nascidos vivos, mas acredita-se que de cada quatro casos apenas um seja relatado (2).

As CGL podem ser divididas clinicamente em quatro grupos: a) CGLl, em que falta tecido adiposo metabolicamente ativo desde o nascimento; b) CGL2, com diminuição tanto de tecido adiposo metabolicamente ativo e de função mecânica desde o nascimento, leve 
retardo mental, cardiomiopatia; c) CGL3, com extrema redução de gordura corporal, baixa estatura e resistência à vitamina $\mathrm{D}$; d) CGL4, com escassez extrema de gordura corporal, miopatia congênita, estenose pilórica e miocardiopatia (4).

A doença se caracteriza por redução do tecido adiposo, extremidades alongadas, aparência acromegálica, crescimento acelerado e cardiomiopatia hipertrófica. É comum a presença de hepatomegalia devida a esteatose, esplenomegalia, acanthosis nigricans, macroglossia, ginecomastia, hiperglicemia, hipertrigliceridemia, hiperinsulinemia, diminuição do HDL-colesterol e elevação do colesterol total e do LDL-colesterol. Caracteristicamente, os níveis séricos de leptina e adiponectina são extremamente baixos (5).

$\mathrm{O}$ manejo da hipertrigliceridemia, especialmente naqueles que manifestam tal alteração ainda na primeira década de vida, é importante na condução clínica dos portadores dessa síndrome. Níveis acima de $500 \mathrm{mg} / \mathrm{dL}$ podem acarretar pancreatite e, em médio e longo prazo, aterosclerose acelerada, esteatose e cirrose hepática $(6,7)$.

Este artigo tem como objetivo ilustrar uma variante clínica de um paciente pediátrico com síndrome lipodistrófica congênita e discutir os riscos e benefícios do uso de bezafibrato em seu tratamento.

\section{MATERIAIS E MÉTODOS}

Foram utilizados dados contidos no prontuário do paciente para compor a apresentação do caso. Os dados referentes ao desenvolvimento estatural do paciente foram computados no software WHO AnthroPlus, disponível em <http://www.who.int/growthref/ tools/en/>, para obtenção da curva de crescimento. Realizou-se pesquisa no banco de dados Medline ${ }^{\circledR} /$ PubMed $^{\circledast}$, utilizando as palavras "bezofibrato/bezafibrate", "fibrato/fibrate", "lactente/infant", "criança/ child/children", assim como buscou-se o termo "Berardinelli syndrome" para elaboração e embasamento das informações presentes no texto.

\section{CASO CLÍNICO}

Criança do sexo masculino, 11 meses de idade, apresentando lipodistrofia generalizada desde o nascimento, com diagnóstico de síndrome de Berardinelli estabelecido por médico geneticista, apresentou-se à consulta endocrinológica com exame de triglicerídeo e coles- terol total séricos de 1.360 e $223 \mathrm{mg} / \mathrm{dL}$, respectivamente. Peso ao nascimento, $3.080 \mathrm{~g}$; estatura, $48 \mathrm{~cm}$. Filho único de pais não consanguíneos, com um caso de lipodistrofia em primo da avó materna falecido aos 24 anos. Mãe e pai com níveis normais de triglicerídeos e ambos com altura de $152 \mathrm{~cm}$. Aleitamento materno exclusivo até os 6 meses de idade, com posterior introdução de alimentação complementar adequada na dieta. Pectus escavatum à ectoscopia. Aparelhos cardiovascular, respiratório e abdome sem outras alterações ao exame físico. Extremidades sem edemas com musculatura muito evidente e de consistência normal. Na idade de 1 ano, estatura 73,5 cm (desvio-padrão $=-1,04$ ); peso $7,4 \mathrm{~kg}$ (desvio-padrão $=-1,7$ para altura), $\mathrm{PA}$ de $85 / 50 \mathrm{mmHg}$. Nessa idade, não foi documentada nenhuma alteração neuropsicomotora.

Possuía valores normais de hemograma, ureia, creatinina, $\mathrm{Na}^{+}, \mathrm{K}^{+}$, cálcio, fósforo, TSH e T4 livre. Demais exames da avaliação inicial e evolução na tabela $l$.

Levando-se em consideração os altos níveis de colesterol e triglicerídeos, foram iniciados aos 11 meses de idade um acompanhamento nutricional adequado e tratamento farmacológico com $30 \mathrm{mg}$ de bezafibrato ao dia $\left(80 \mathrm{mg} / \mathrm{m}^{2}\right.$ de superfície corporal). Foi explicado aos pais o caráter de excepcionalidade da adoção da medida farmacológica, tendo a mãe assinado TCLE autorizando a instituição da terapia medicamentosa.

Com o início do uso de bezafibrato, o paciente apresentou queda significativa de triglicerídeos séricos, que se mantiveram entre valores de 55 a $536 \mathrm{mg} / \mathrm{dL}$ (valor antes do tratamento: $1.360 \mathrm{mg} / \mathrm{dL}$ ).

A curva de crescimento do paciente pode ser visualizada no gráfico 1 (estatura-alvo do paciente $158,5 \mathrm{~cm}$ ).

$\mathrm{Na}$ idade de 1 ano e 9 meses, a mãe relatou dificuldade no desenvolvimento da fala, sendo então recomendada avaliação neuropediátrica e fonoaudiológica. Aos 3 anos e 10 meses ainda não elaborava frases completas.

Atualmente, o paciente tem estigmas clássicos da síndrome (Figura 1) e apresenta dificuldade na fala, mas não apresenta sinais de desenvolvimento de caracteres sexuais secundários (Tanner MlPl). Aos 5 anos e 5 meses, apresenta idade óssea de 5 anos. Confirmou-se a redução acentuada de tecido adiposo mecânico por meio de tomografia computadorizada de órbita (Figura 2). Permanece em uso de bezafibrato e realiza seguimento nutricional. TCLE foi assinado pelo responsável (mãe) para publicação de imagens. 
Tabela 1. Dose de bezafibrato e evolução laboratorial durante os primeiros 5 anos e meio de vida

\begin{tabular}{|c|c|c|c|c|c|c|c|c|c|}
\hline Parâmetros IC* & $11 \mathrm{~m}$ & 1 ano $3 \mathrm{~m}$ & 2 anos $4 m$ & $\begin{array}{c}3 \text { anos } \\
1 \mathrm{~m}\end{array}$ & $\begin{array}{c}3 \text { anos } \\
10 \mathrm{~m}\end{array}$ & 4 anos $3 \mathrm{~m}$ & 4 anos $5 \mathrm{~m}$ & 5 anos $3 \mathrm{~m}$ & 5 anos $5 \mathrm{~m}$ \\
\hline Dose de Bezafibrato (mg) & 30 & 30 & 35 & 50 & 60 & 30 & 50 & 55 & 55 \\
\hline Glicemia (mg/dl) & 86 & 68 & 68 & 88 & - & 89 & - & 55 & 66 \\
\hline $\begin{array}{l}\text { Hemoglobina } \\
\text { glicada-A1c (\%) }\end{array}$ & - & - & 6,1 & - & - & - & - & - & 5,7 \\
\hline Insulina (mU/mL) & - & - & - & 2,9 & 5,5 & - & - & 11,3 & 7,3 \\
\hline Gama-GT (UI) & - & - & - & 26 & - & - & - & - & - \\
\hline Colesterol (mg/dL) & 223 & 169 & 171 & 178 & 142 & 162 & - & 136 & 135 \\
\hline LDL-colesterol (mg/dL) & - & 76 & 124 & 113 & 91 & 99 & - & 67 & - \\
\hline HDL-colesterol (mg/dL) & - & 39 & 35 & 41 & 40 & 39 & - & 32 & 29 \\
\hline Triglicerides (mg/dL) & 1360 & 270 & 62 & 121 & 55 & 118 & - & 182 & 406 \\
\hline CPK (U/I) & - & 176 & - & 112 & 516 & 332,8 & 189 & 93 & 179 \\
\hline AST (U/I) & - & 28 & - & 35 & - & 41 & - & - & 43 \\
\hline ALT (U/l) & - & 33 & - & 34 & - & 70 & - & - & 53 \\
\hline $\begin{array}{l}\text { Ultrassonografia } \\
\text { abdominal }\end{array}$ & $\begin{array}{c}\text { Sem } \\
\text { alterações }\end{array}$ & - & $\begin{array}{c}\text { Esteatose } \\
\text { hepática } \\
\text { leve }\end{array}$ & - & $\begin{array}{c}\text { Esteatose } \\
\text { hepática } \\
\text { leve }\end{array}$ & - & - & $\begin{array}{c}\text { Esteatose } \\
\text { hepática leve e } \\
\text { hepatomegalia } \\
\text { discreta }\end{array}$ & - \\
\hline Ecocardiograma & - & $\begin{array}{c}\text { Leve } \\
\text { perviedade } \\
\text { de forame } \\
\text { oval }\end{array}$ & - & - & - & $\begin{array}{l}\text { Leve } \\
\text { perviedade } \\
\text { de forame } \\
\text { oval }\end{array}$ & - & - & $\begin{array}{l}\text { Escape } \\
\text { valvar } \\
\text { tricúspide } \\
\text { pulmonar }\end{array}$ \\
\hline
\end{tabular}

*Idade cronológica.

WHO child growth standards (birth to 60 months), WHO reference 2007 (61 months to 19 years)

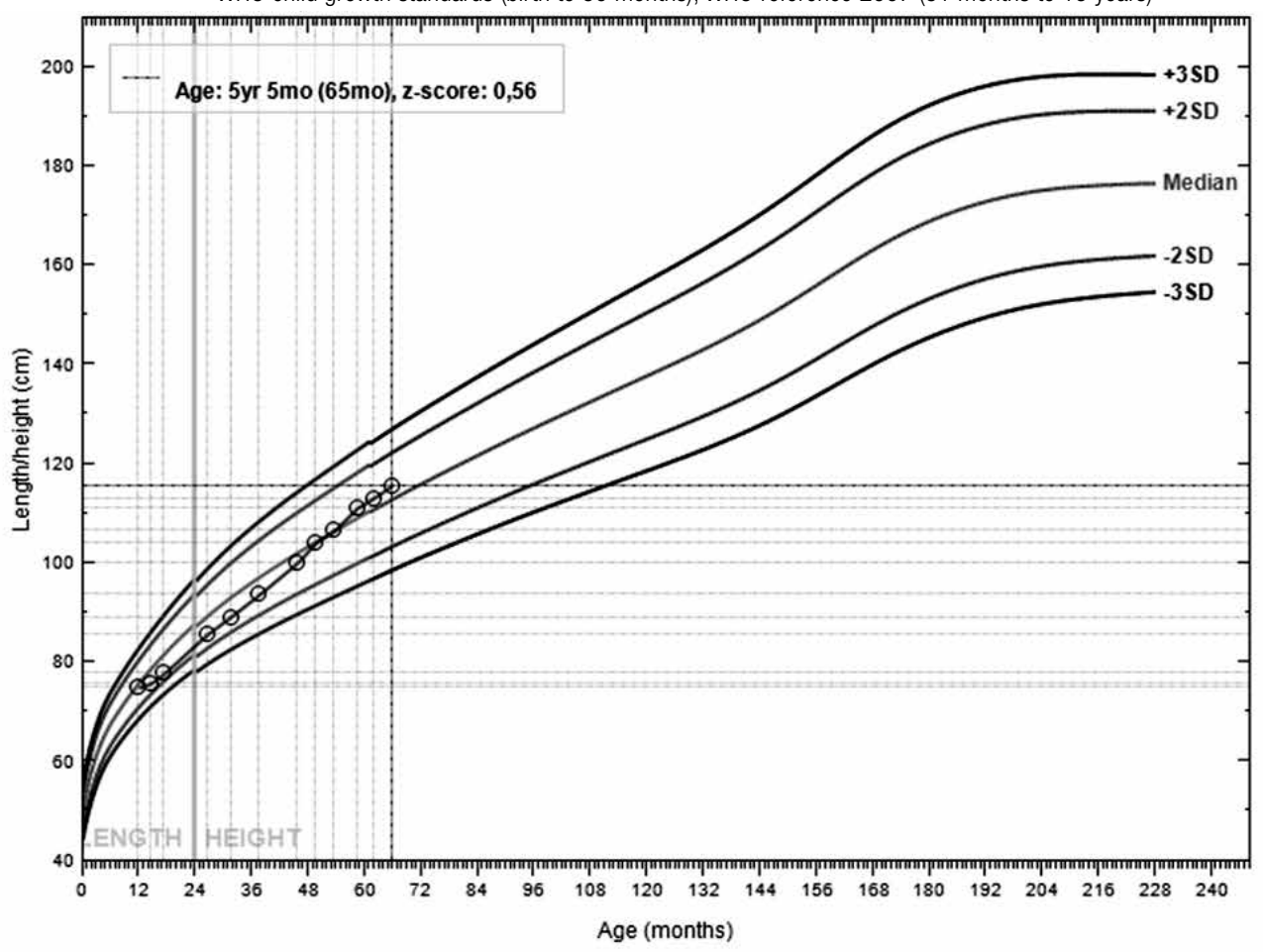

Gráfico 1. Curva de altura versus idade. 


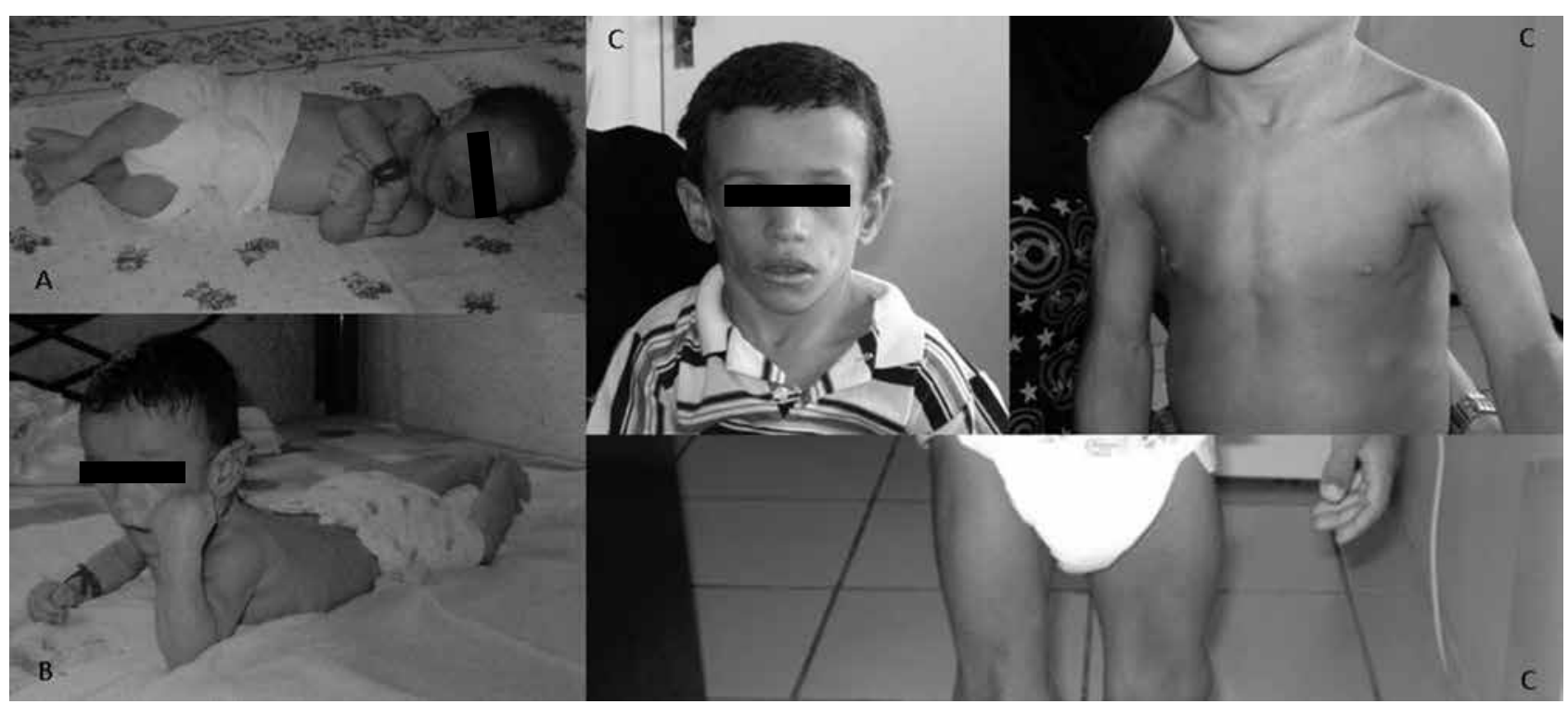

Figura 1. Aspectos clínicos do paciente demonstrando fácies triangular, hipertrofia muscular, lipoatrofia generalizada, língua protrusa, mãos e pés proeminentes. (A) Paciente com 1 mês de vida; (B) aos 5 meses; (C) aos 5 anos e 5 meses.

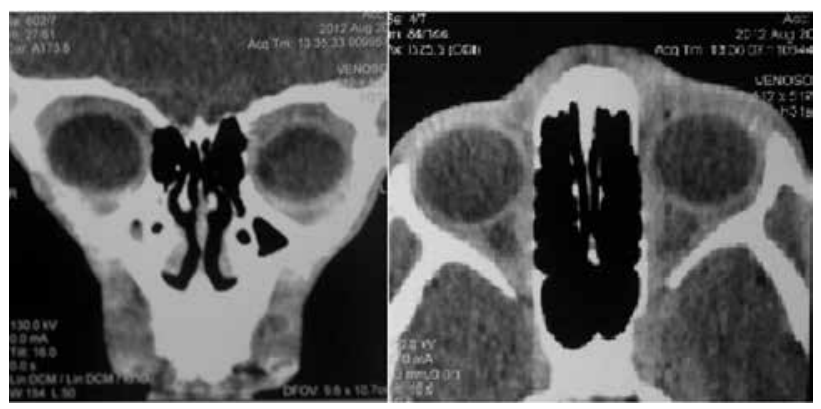

Figura 2. Imagem de tomografia computadorizada do paciente evidenciando acentuada redução da gordura orbitária.

\section{DISCUSSÃO}

Lipodistrofia deve ser considerada no diagnóstico diferencial de pacientes com hipertrofia muscular, diabetes precoce, hipertrigliceridemia severa, esteatose hepática, hepatoesplenomegalia, acanthosis nigricans e cardiomiopatia. Em nosso relato, aos 11 meses, a criança foi diagnosticada com lipodistrofia generalizada e hipertrigliceridemia grave.

Nosso paciente pode ser clinicamente enquadrado em CGL2, que, segundo Garg (4), possui maior prevalência da mutação em BSCL2, pois se apresentou precocemente com lipodistrofia generalizada desde o nascimento (Figuras 1 e 2) associada a retardo mental e cardiopatia. Pacientes com mutações BSCL2 têm a variedade mais grave da CGL e nascem com intensa redução de gordura corporal. Nesses casos, diabetes, hiperlipidemia e esteatose hepática representam um desafio terapêutico. Os pacientes podem desenvolver ne- fropatia e retinopatia diabética, ataques recorrentes de pancreatite aguda e cirrose, que ocasionalmente são as causas de mortalidade (4,7-10).

A hipertrigliceridemia é um achado frequente na CGL, especialmente nos portadores de mutações do gene BSCL2. Nesses casos, a dosagem sérica de triglicerídeos pode chegar a níveis muito elevados como os observados nesse lactente de 11 meses de idade, que apresentou um valor de $1.360 \mathrm{mg} / \mathrm{dL}$. Entretanto, são escassos os relatos de casos com valores acima de $1.000 \mathrm{mg} / \mathrm{dL}$ nessa faixa etária. Durante a revisão, observou-se apenas um caso de uma criança indiana com nível de triglicerídeos que estava próximo do aqui relatado, sendo o valor descrito de $1.391 \mathrm{mg} / \mathrm{dL}$ aos 5 meses de idade. Nesse caso, apesar de o controle glicêmico com insulina NPH controlar a glicemia, a criança evoluiu com acidente vascular cerebral (AVC) isquêmico aos 8 meses; o nível mínimo atingido de triglicerídeos foi de $800 \mathrm{mg} / \mathrm{dL}$ com ajuste da dieta e uso de ômega-3 (11).

As tendências atuais para o manejo de crianças com dislipidemia defendem fortemente as mudanças do estilo de vida como via terapêutica primária, incluindo redução da ingestão de gorduras saturadas, colesterol, ácidos graxos de cadeia longa, açúcar refinado, aumento do consumo de gorduras poli-insaturadas, carboidratos complexos e proteínas solúveis, bem como aumento da atividade física. A terapia com drogas deve ser considerada nas formas mais graves de hipertrigliceridemia, bem como em indivíduos nos quais as mudanças do 
estilo de vida não obtiveram eficácia terapêutica em diminuir os triglicerídeos (12).

Em virtude da escassez de dados disponíveis para pacientes pediátricos, a extrapolação de recomendações para manejo de hipertrigliceridemia em adultos pode ser considerada, tendo os fibratos como agentes farmacológicos primários (13). Os fibratos são medicamentos derivados do ácido fíbrico com mecanismos de ação complexos, que envolvem estimulação de receptores nucleares PPAR- $\alpha$, indução da captação hepática de ácidos graxos, aumento de sua conversão em acetil-CoA e inibição da lipase hormônio-sensível no tecido adiposo. Dessa forma, há redução da produção, da liberação e dos níveis séricos dos triglicerídeos $(14,15)$. Também aumentam os níveis de HDL-C e podem, ainda, causar diminuição nos níveis de LDL-C (14-16).

Em geral, essas drogas são consideradas bem toleradas, com excelente perfil de segurança e baixa incidência de toxicidade relatada (17). Os principais efeitos colaterais incluem sintomas gastrointestinais (sobretudo náuseas e diarreia), redução da libido, dores musculares, astenia, erupções cutâneas, prurido, cefaleia, insônia e elevação discreta das transaminases e creatinoquinases (18). Interações clinicamente relevantes de fibratos com outros antilipidêmicos incluem miopatia e rabdomiólise, principalmente quando combinado ao uso de estatinas, e diminuição da biodisponibilidade quando combinados com outros sequestradores de ácido biliar. Fibratos também podem potencializar o efeito de anticoagulantes cumarínicos, podendo causar sangramentos (19).

A dose do bezafibrato recomendada para pacientes pediátricos varia de 10 a $20 \mathrm{mg} /$ dia (13). Com essa dose, Wheeler e cols. (20) estudaram durante seis meses 14 crianças de 4 a 15 anos de idade portadoras de hipercolesterolemia familial e observaram redução de $22 \%$ e $23 \%$ nas concentrações de colesterol total e triglicérides no plasma, respectivamente. Nenhum efeito colateral foi observado durante o período de observação. Em nosso paciente foi utilizada dose de $30 \mathrm{mg} /$ dia - correspondente a aproximadamente $80 \mathrm{mg} / \mathrm{m}^{2}$ - por causa do caráter excepcional da trigliceridemia e da associação com a lipodistrofia. Essa classe de drogas pode ser usada preferencialmente em crianças com elevação grave de triglicerídeos e com alto risco de desenvolver pancreatite (14-16). Não foi encontrada descrição de uso de fibrato em lactente. O relato do menino indiano já mencionado foi o único encontrado com hipertrigliceridemia em níveis e faixa etária semelhante aos do nosso paciente. Naquele, o uso exclusivo de ômega-3 e insulina conseguiu um nadir de apenas $800 \mathrm{mg} / \mathrm{dL}$, e o paciente evoluiu com AVC isquêmico aos 8 meses (11). Em nosso relato, o menor valor de triglicerídeos foi de $55 \mathrm{mg} / \mathrm{dL}$ aos 3 anos e 10 meses, quando o paciente fez uso da maior dose ao longo do tratamento, $60 \mathrm{mg} /$ dia $\left(100 \mathrm{mg} / \mathrm{m}^{2}\right)$. Observa-se que a dose variou de $30 \mathrm{a}$ $60 \mathrm{mg} / \mathrm{dia}$, dose acima da indicada nos casos de hipertrigliceridemia extrema não associada à lipodistrofia (14).

Em virtude do aumento do risco de miopatia por uso dessa droga, é recomendado o acompanhamento dos níveis de CPK. De acordo com o observado, houve aumento dos níveis de CPK aos 3 anos de idade (dois anos de uso de bezafibrato). É importante observar que essa criança apresentou elevação da enzima muscular em um período da infância que se caracteriza por aumento das atividades físicas não supervisionadas, predispondo a pequenos traumas, que também podem ser causas de elevações modestas e transitórias de CPK. Pensando na implicação do medicamento nessa situação, a diminuição da dose foi realizada, seguida de normalização laboratorial de CPK.

Avaliação antropométrica no segundo ano de vida evidenciou velocidade de crescimento de $20 \mathrm{~cm} / \mathrm{ano}$, o dobro do esperado para população pediátrica em geral. Do terceiro ao quinto ano, o paciente obteve 9,3 $\mathrm{cm} / \mathrm{ano}$, com diferença crescente do desvio-padrão, mostrando inclusive um padrão diferente da população pediátrica saudável, que é de estabilidade em níveis menores $(5-7 \mathrm{~cm} / \mathrm{ano})$. Não há descrições do perfil de crescimento de pacientes com CGL segundo genotipagem ou grupos clínicos. Logo, a comparação desse paciente com a população geral permite supor que essa diferença seria resultado dos distúrbios metabólicos da síndrome, principalmente da resistência insulínica. $\mathrm{O}$ próprio crescimento exacerbado poderia ser um dos sinais mais precoces do desenvolvimento pleno de resistência insulínica, mesmo quando os índices laboratoriais ainda não se encontram alterados.

\section{CONCLUSÃO}

Portadores de CGL, principalmente do tipo CGL2, têm maior risco de desenvolver intensa hipertrigliceridemia muito precocemente, como observado no paciente relatado. Neste caso, mesmo não havendo recomendações formais para uso na faixa etária inicial do paciente, o bezafibrato foi bem tolerado. A dose de 80 a $100 \mathrm{mg} / \mathrm{m}^{2}$ manteve os níveis de triglicerídeos 
em faixa aceitável, mostrando eficácia na diminuição de triglicerídeos séricos. Como efeito adverso ocorreu apenas elevação dos níveis de $\mathrm{CPK}$, que apresentou regressão após manejo da dose. Com prosseguimento do tratamento, esses níveis mantiveram-se estáveis. O manejo clínico da hipertrigliceridemia com bezafibrato - realizando-se seguimento clínico-laboratorial regular - apresentou-se eficaz e seguro no período descrito. No entanto, são necessários mais estudos para garantir a eficácia e a segurança em longo prazo do uso de bezafibrato na faixa etária pediátrica.

Agradecimentos: a todos os participantes do Núcleo de Estudos em Endocrinologia da UESPI, por participarem dos debates sobre os questionamentos levantados durante a elaboração no presente relato de caso.

Declaração: os autores declaram não haver conflitos de interesse científico neste estudo.

\section{REFERÊNCIAS}

1. Garg A. Lipodystrophies. Am J Med. 2000;108:143-52.

2. Garg A. Medical progress: acquired and inherited lipodystrophies. N Engl J Med. 2004;350(12):1220-34.

3. Garg A, Misra A. Lipodystrophies: rare disorders causing metabolic syndrome. Endocrinol Metab Clin North Am. 2004;33:305-31.

4. Garg AJ. Clinical review\#: Lipodystrophies: genetic and acquired body fat disorders. Clin Endocrinol Metab. 2011;96(11):3313-25.

5. Haque WA, Shimomura I, Matsuzawa $Y$, Garg A. Serum adiponectin and leptin levels in patients with lipodystrophies. J Clin Endocrinol Metab. 2002;87(5):2395.

6. Yadav D, Pitchumoni CS. Issues in hyperlipidemic pancreatitis. J Clin Gastroenterol. 2003;36(1):54-62.

7. Simha V, Garg A. Inherited lipodystrophies and hypertriglyceridemia Curr Opin Lipidol. 2009;20(4):300-8.
8. SimhaV, Garg A. Phenotypic heterogeneity in body fat distribution in patients with congenital generalized lipodystrophy due to mutations in the AGPAT2 or Seipin genes. J Clin Endocrinol Metab. 2003;88:5433-7.

9. Simha V, Agarwal AK, Aronin PA, lannaccone ST, Garg A. Novel subtype of congenital generalized lipodystrophy associated with muscular weakness and cervical spine instability. Am J Med Genet A. 2008;146A:2318-26.

10. Simha V, Garg A. Inherited lipodystrophies and hypertriglyceridemia. Curr Opin Lipidol. 2009;20(4):300-8.

11. Indumathi CK, Lewin S, Ayyar V. Berardinelli Seip syndrome with insulin-resistant diabetes mellitus and stroke in an infant. Indian J Endocrinol Metab. 2011;15(Suppl 1):S62-4.

12. McCrindle BW, Urbina EM, Dennison BA, Jacobson MS, Steinberger J, Rocchini AP, et al. Drug therapy of high-risk lipid abnormalities in children and adolescents: a scientific statement from the American Heart Association Atherosclerosis, Hypertension, and Obesity in Youth Committee, Council of Cardiovascular Disease in the Young, with the Council on Cardiovascular Nursing. Circulation. 2007;115(14):1948-67.

13. Castro PSG, Oliveira FLC. Prevenção da aterosclerose e tratamento medicamentoso de anormalidades lipídicas de alto risco em crianças e adolescentes. J Pediatr (Rio J). 2009;85(1):6-14.

14. Yuan G, Al-Shali KZ, Hegele RA. Hypertriglyceridemia: its etiology, effects and treatment. CMAJ. 2007;176(8):1113-20.

15. Martin G, Schoonjans K, Lefebvre A, Staels B, Auwerx J. Coordinate regulation of the expression of the fatty acid transporter protein (FATP) and acyl CoA synthetase (ACS) genes by PPARa and PPARg activators. J Biol Chem. 1997;272:28210-7.

16. Newman T, Hulley S. Carcinogenicity of lipid-lowering drugs. JAMA. 1996;275:55-60.

17. Sgro C, Escousse A. Side effects of fibrates (except liver and muscle). Therapie. 1991;46:351-4.

18. Remick J, Weintraub $H$, Setton R, Offenbacher J, Fisher E, Schwartzbard A. Fibrate therapy: an update. Cardiol Rev. 2008;16:129-41.

19. Blum A, Seligmann H, Livneh A, Ezra D. Severe gastrointestinal bleeding induced by a probable hydroxycoumarin-bezafibrate interaction. Isr J Med Sci. 1992;28:47-9.

20. Wheeler KA, West RJ, Lloyd JK, Barley J. Double blind trial of bezafibrate in familial hypercholesterolaemia. Arch Dis Child. 1985;60(1):34-7. 